\title{
Coherent optical vortices from relativistic electron beams
}

\author{
Erik Hemsing ${ }^{1 \star}$, Andrey Knyazik ${ }^{2}$, Michael Dunning ${ }^{1}$, Dao Xiang $^{1}$, Agostino Marinelli ${ }^{1}$, Carsten Hast ${ }^{1}$ \\ and James B. Rosenzweig ${ }^{2}$
}

Recent advances in the production and control of highbrightness electron beams (e-beams) have enabled a new class of intense light sources based on the free electron laser (FEL) that can examine matter at ångstrom length and femtosecond time scales ${ }^{1}$. The free, or unbound, electrons act as the lasing medium, which provides unique opportunities to exquisitely control the spatial and temporal structure of the emitted light through precision manipulation of the electron distribution. We present an experimental demonstration of light with orbital angular momentum (OAM; ref. 2) generated from a relativistic e-beam rearranged into an optical scale helix by a laser. With this technique, we show that a Gaussian laser mode can be effectively up-converted to an OAM mode in an FEL using only the e-beam as a mode-converter. Results confirm theoretical predictions ${ }^{3,4}$, and pave the way for the production of coherent OAM light with unprecedented brightness down to hard $X$-ray wavelengths for wide ranging applications in modern light sources.

Light beams that carry OAM have become the subject of intense interest for numerous applications ${ }^{5}$, including particle micromanipulation ${ }^{6}$, microscopy $^{7}$, imaging ${ }^{8}$, optical pump schemes ${ }^{9}$, quantum entanglement ${ }^{10}$, and communications ${ }^{11}$. As first shown by Allen et al., ${ }^{2}$, these beams have a doughnut-shaped intensity profile and carry discrete values $l \hbar$ of OAM per photon as a result of the $\mathrm{e}^{\mathrm{i} l \phi}$ dependence of the complex field, where $\phi$ is the azimuthal coordinate and $l$ is an integer referred to as the topological charge.

The multitude of emerging applications enabled by OAM light at visible and longer wavelengths suggests new research opportunities in the extreme ultraviolet (EUV) to hard X-ray regime where a welldefined OAM provides an additional degree of freedom that may be specifically exploited to probe the deep structure and behaviour of matter. Promising applications include expanded X-ray magnetic circular dichroism ${ }^{12}$, where angle-resolved energy loss spectrometry distinguishes spin-polarized atomic transitions subject to different photon OAM and polarization states ${ }^{13}$. Traditionally, these 'optical vortices' are created by shaping of the phase front of a laser as it passes through different optical media ${ }^{14-16}$, such as spiral phase plates ${ }^{17}$ or computer generated holograms ${ }^{18}$. Analogous techniques have also been used to transform X-rays into vortices at synchrotron light sources ${ }^{19,20}$, and alternative methods suggest vortex beams can be created through Compton back-scattering ${ }^{21}$ or harmonic emission in undulators ${ }^{22}$. Here, we report on a completely different technique in which a simple Gaussian laser pulse is used to generate fully coherent $l=1$ OAM light purely through its interaction with a relativistic electron beam (e-beam).

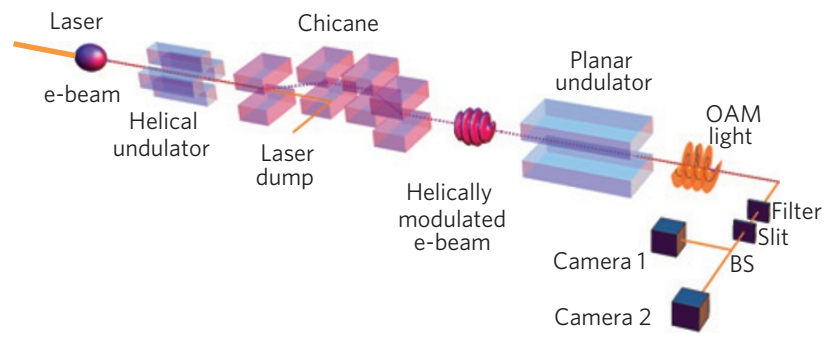

Figure 1 | Illustration of the experiment (not to scale). The unmodulated relativistic electron beam interacts with a linearly polarized laser in a helical undulator, which gives the electrons an energy kick that depends on their position in the focused laser beam. The e-beam then traverses a longitudinally dispersive chicane that allows the electrons with higher energy to catch up to those with lower energy (momentum compaction). The result is a 'helically microbunched' beam that then radiates light with $\mathrm{OAM}$ at the fundamental frequency in the planar undulator.

By using the e-beam as the lossless medium, this principle of in situ mode-conversion enables coherent OAM production in modern FELs, which can access a virtually unlimited range of wavelengths, produce femtosecond pulses, and generate $\mathrm{X}$-rays with ten orders of magnitude higher brightness than previous sources ${ }^{1}$. More generally, this technique illustrates the emerging concept of 'beam by design' in advanced accelerator-based light sources, where the electron beam can be precisely manipulated with lasers to radiate precision tailored light.

Light in an FEL is produced by a relativistic e-beam traversing a periodic magnetic undulator (radiator). The emission wavelength $\lambda=2 \pi / k$ is given by

$$
\lambda=\frac{\lambda_{u}}{2 n_{\mathrm{h}} \gamma^{2}}\left(1+K^{2}\right)
$$

where $\lambda_{u}$ is the undulator period, $\gamma=E / m c^{2}$ the relativistic factor of the electron with energy $E$ and mass $m, n_{\mathrm{h}}$ the harmonic number, and $K$ the normalized strength of the undulator. Intense coherent light is emitted from an e-beam that is microbunched, wherein the electrons are piled-up at the emission wavelength. In an FEL the electrons are initially distributed randomly, so microbunching occurs either as a result of the FEL instability (where the amplified light acts back on the beam to rearrange the electrons), or by way of an external laser acting on the beam upstream of the FEL. In either case, the emitted radiation

${ }^{1}$ SLAC National Accelerator Laboratory, Menlo Park, California 94025, USA, ${ }^{2}$ Department of Physics and Astronomy, University of California Los Angeles, Los Angeles, California 90095, USA. *e-mail: ehemsing@slac.stanford.edu 


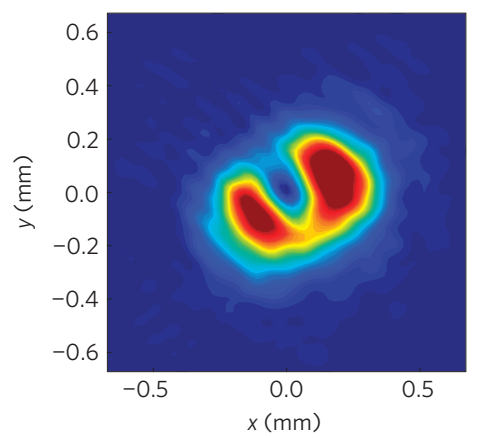

c

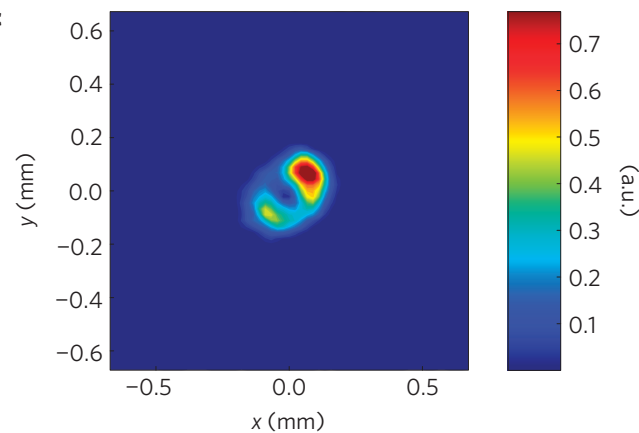

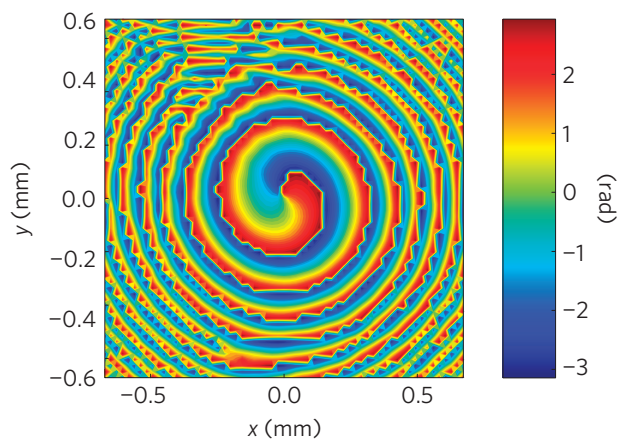

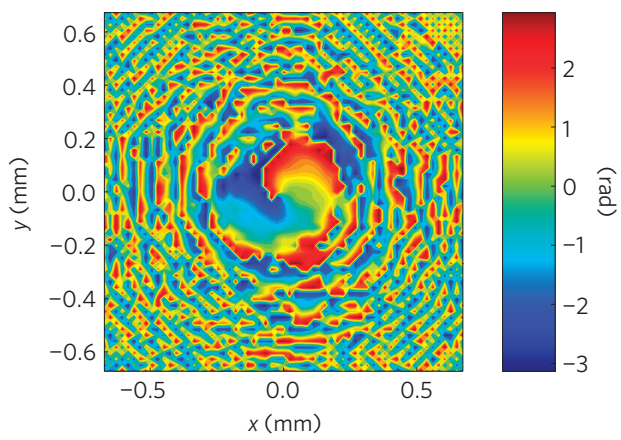

Figure 2 | Measured undulator radiation intensities (left) and reconstructed $I=1$ OAM phases (right) from two cameras positioned to view the undulator radiation profiles at different planes. a-d, Intensity (a) and phase (b) at camera 1 are shown, and correspond to the intensity (c) and phase (d) at camera 2.

distribution depends both on the microbunching structure and on the angular and spectral emission geometry of the FEL radiator. Thus, because the radiation at the dominant fundamental wavelength $\left(n_{\mathrm{h}}=1\right)$ of an FEL is strongly peaked on axis, the unique phase structure of OAM light must originate from a helically microbunched beam in which the electrons are concentrated in a matching spiral-staircase-like distribution. This fine-tuned structure demands precision three-dimensional manipulation of the electron distribution upstream of the FEL, on the scale of a single wavelength.

It turns out this beam structure can arise naturally through a specific laser interaction. The technique was first proposed in ref. 3 and was later examined at low beam energies and mid-infrared wavelengths ${ }^{23}$, but direct measurement of the unique transverse OAM structure was not available. An expanded scheme specifically applicable to modern FELs and relevant to this work was then proposed in ref. 4. The concept relies on a harmonic interaction between the e-beam and a seed laser in a circularly polarized undulator to naturally produce a helical energy modulation in the electron distribution. After dispersion through transport, the modulation is then converted into helical microbunching where the screw-like beam distribution emits and amplifies coherent OAM light in a downstream undulator.

\section{Experiment and method}

In such seeded FEL configurations, an important issue for the production of OAM light is the mode content of the electron distribution. The correlated helical structure must exceed the intrinsic shot noise distribution so that the OAM light dominates, and it must survive transport to the radiator to preserve the mode purity. Here we experimentally examine both of these issues by direct measurement of the emitted OAM light. The set-up is shown in Fig. 1, which illustrates our experimental configuration at the SLAC Next Linear Collider Test Accelerator (refs 24,25). The initially unmodulated $120 \mathrm{MeV}$ e-beam ( $\gamma=235,0.5$ ps FWHM duration) interacts with the $\lambda=800 \mathrm{~nm}$ transversely Gaussian laser pulse in the helical undulator (modulator) with periodicity $\lambda_{u}=5.26 \mathrm{~cm}$ and strength $K=1.52$. From equation (1), this excites an energy modulation at the wavelength $\lambda$ in the e-beam at the second harmonic resonance. The energy modulation has a helical spatial structure as a consequence of the three-dimensional harmonic interaction geometry. Modulations of the form $\mathrm{e}^{\mathrm{i} k z-\mathrm{i} l \phi}$ in the beam are predicted at the harmonic $n_{\mathrm{h}}$ according to

$$
l= \pm\left(n_{\mathrm{h}}-1\right)
$$

where the upper $(+)$ sign is taken for our right-circularly polarized modulator. The linearly polarized laser field profile $E_{l}(r)=E_{0} \mathrm{e}^{-r^{2} / w_{0}^{2}}$ excites a right-handed $l=1$ helical modulation at the second harmonic, given by

$$
\Delta \gamma(r, \phi, s)=\frac{q K^{2} N_{u} \lambda_{u}^{2}}{8 \pi \gamma^{2} m c^{2}} \frac{\partial E_{l}(r)}{\partial r} \cos (k s-\phi)
$$

where $N_{u}=4$ is the number of modulator periods, $s$ is the longitudinal position of an electron, and $q$ the electron charge. The coupling is proportional to the radial variation of the Gaussian laser field, so electrons on axis $(r=0)$ are unmodulated, whereas those at the radial position $r_{\max }=w_{0} / \sqrt{2}$ and helical position $k s-\phi=n \pi$ receive the largest kick. In the experiment, the laser spot size was $w_{0}=290 \mu \mathrm{m}$ and the e-beam $\left(N=3 \times 10^{8}\right.$ electrons) was focused to a root mean square spot size $w_{0} / 2=$ $145 \mu \mathrm{m}$ to maximize both the laser overlap and bunching factor according to predictions ${ }^{4}$.

The subsequent dispersive magnetic chicane, characterized by the matrix transport element $R_{56}=\gamma \partial s / \partial \gamma=1.9 \mathrm{~mm}$ converts the energy modulation into a helical density modulation. The electrons follow an energy-dependent path through the chicane that modifies their relative longitudinal positions according to 


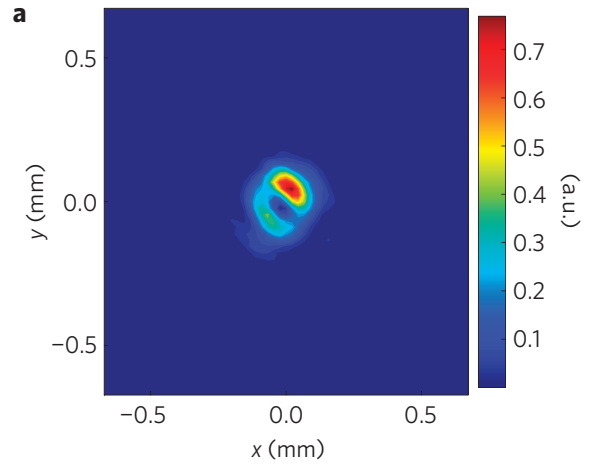

d

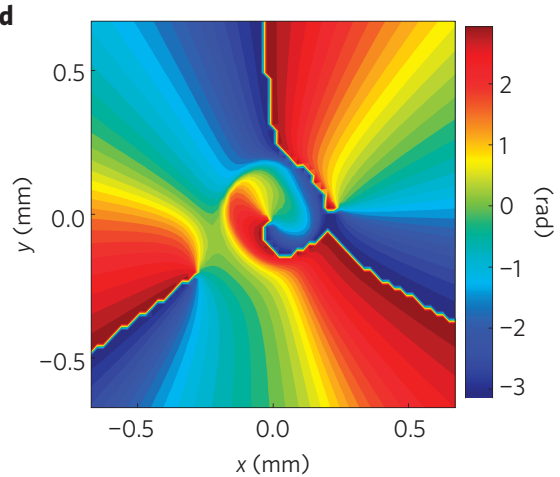

b

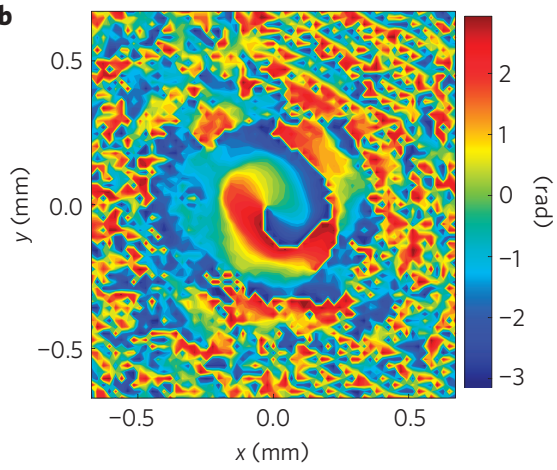

e

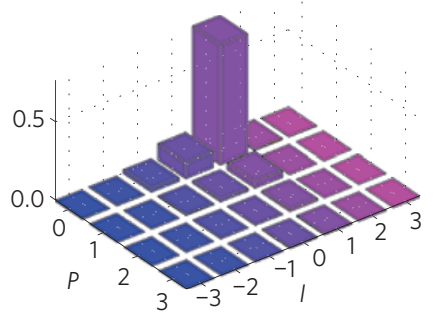

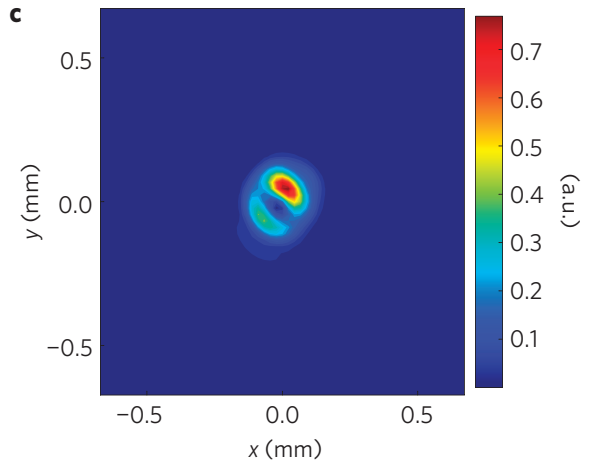

f

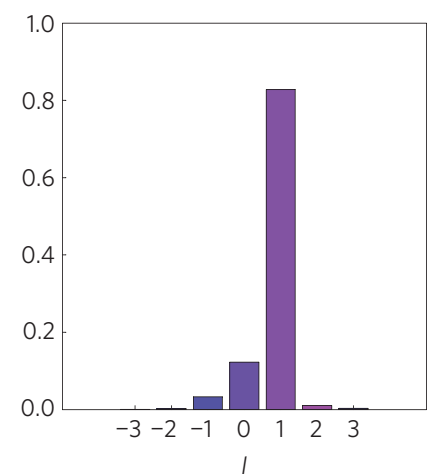

Figure $\mathbf{3}$ | Complex field profile calculated at the undulator exit. a,b, Field intensity (a) and phase (b) obtained from the phase retrieval algorithm, propagated back to the undulator. $\mathbf{c}, \mathbf{d}$, Field intensity (c) and phase (d) expressed as a superposition of orthogonal radial $p$ and azimuthal / modes with the amplitudes $a_{p, I}$ given in $\mathbf{e}$. The expansion accurately reproduces the OAM amplitude and phase in the relevant peak-intensity region. The total power in the $\mathrm{OAM} /$ modes is found by summing over $p(\mathbf{f})$. The expansion parameter is $a=180 \mu \mathrm{m}$.
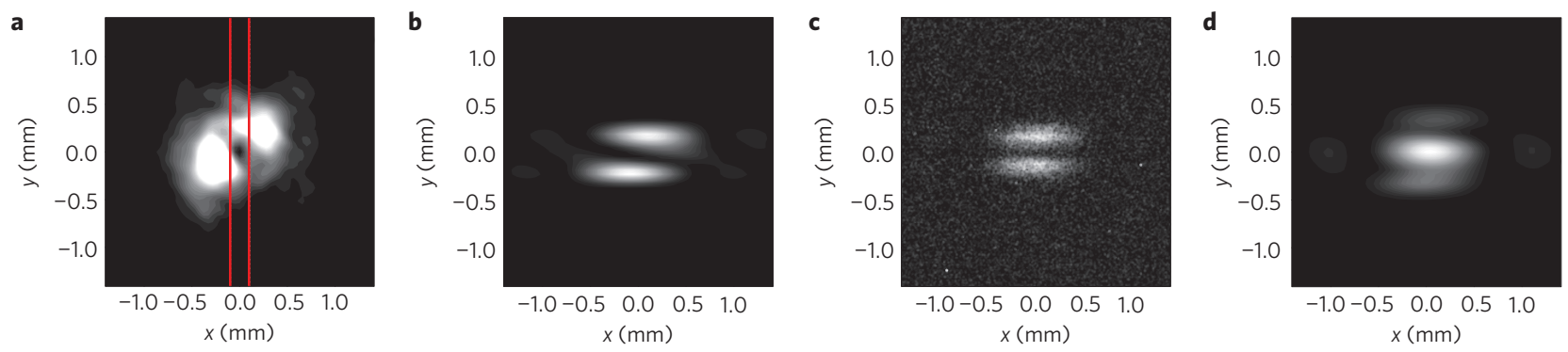

Figure 4 | Diffraction pattern of OAM mode through a vertical slit. a, Vertical red lines show the slit size and position centred on the phase singularity of the calculated OAM mode at the slit. $\mathbf{b}$, The calculated radiation profile at camera 2 shows a null on-axis characteristic of the helical phase. $\mathbf{c}$, This closely matches the features observed in the experimentally measured pattern. $\mathbf{d}$. Diffraction pattern of the intensity in a with a flat phase front, shown for comparison with $\mathbf{b}, \mathbf{c}$.

$s \rightarrow s+R_{56} \Delta \gamma / \gamma$. At the chicane exit, the bunching factor is then given by the sum $b_{l}=N^{-1} \sum_{j}^{N} \mathrm{e}^{\mathrm{i} k s_{j}-i l \phi_{j}}$. Bunching into the $l=1$ mode is maximized when electrons with the largest energy modulation move longitudinally by $\sim \lambda / 4$, so the condition $R_{56} \Delta \gamma\left(r_{\max }\right) / \gamma \simeq$ $\lambda / 4$ sets the optimal laser field and dispersion. The helically microbunched beam then radiates coherently in the 10-period planar undulator, which is tuned to emit light at $800 \mathrm{~nm}$ at the $n_{\mathrm{h}}=1$ fundamental wavelength $\left(\lambda_{w, r}=3.3 \mathrm{~cm}, K_{r}=1.29\right)$. As a result, $l=1$ OAM light at $800 \mathrm{~nm}$ is produced without phase front manipulation by external optics from an $800 \mathrm{~nm}, l=0$ laser mode acting on the e-beam.

The linearly polarized laser ( $35 \mu \mathrm{J}, 1 \mathrm{ps}$ FWHM) pulse was injected through a polarizer to control the laser field strength in the modulator. Only incoherent undulator radiation was visible with the polarizer set to full laser attenuation, but coherent OAM light $130 \times$ more intense was readily observed with the polarizer rotated by 2 degrees. This corresponds to a field strength of $E_{0}=15 \mathrm{MV} \mathrm{m}^{-1}$ at the laser waist in the modulator, yielding an energy modulation amplitude of $\sim 0.8 \mathrm{keV}$ (about half the slice energy spread). Given these parameters, theory predicts coherent bunching in the $l=1$ mode of $\sim 3 \%$ - significantly larger than the $\mathcal{O}\left(10^{-4}\right)$ intrinsic shot noise in modern FELs, and thus more than enough to seed dominant exponential OAM mode growth in these devices. Calculations indicate bunching up to $b_{1} \sim 50 \%$ is reached in our set-up at 30 degrees rotation $(9 \mu \mathrm{J}$ input).

Light emitted by the e-beam in the planar undulator was sent through a $800 \pm 5 \mathrm{~nm}$ bandpass filter and then through a beam splitter to two identical cameras, which simultaneously record the intensity profile at two downstream locations (see Fig. 1). Camera 1, with a lens of effective focal length $7.0 \mathrm{~cm}$, was set to image the 
extraction mirror in the beamline $21 \mathrm{~cm}$ away. Camera 2 (effective focal length $10.8 \mathrm{~cm}$ ) recorded the far-field profile distribution with the focus set to infinity. The pair of intensity profile images (Fig. 2a,c) was then processed with an iterative phase retrieval algorithm ${ }^{26}$. Similar to standard techniques, this routine exploits the defocus variation and Fourier-transformation properties of transport between image planes to reconstruct the phase from the known intensities ${ }^{27}$. For each iteration cycle, the sum squared error between the new amplitudes and the measured amplitudes is checked, and the algorithm continues until it converges or stagnates. Stagnation occurs between 200 and 500 iterations, depending on the initial guess for the phase (either flat everywhere or random noise). Shown in Fig. 2b,d, the reconstructed phases show the presence of a dominant optical vortex with an $l=1$ right-handedness, consistent with equation (2).

The slight ellipticity in the observed intensity patterns indicates the presence of other, weaker optical modes in the radiation pattern, probably due to imperfect roundness of the e-beam and/or the excitation of adjacent modes in the modulator from experimental asymmetries $^{23}$. In our experiment this structure actually improves the robustness of the reconstruction algorithm because optical dispersion of multiple modes during propagation results in an observable rotation in the intensity profile at different distances. A dominant $l=-1$ mode would lead to rotation in the opposite direction, so the algorithm converges reliably to the $l=1$ mode even in the presence of initial random phase noise.

To ascertain the purity and relative power contained in the dominant OAM mode, the reconstructed complex fields were decomposed into an orthogonal cylindrical mode basis of LaguerreGaussian (LG) beams,

$$
E_{u}(r, \phi)=C \sum_{p=0}^{\infty} \sum_{l=-\infty}^{\infty} a_{p, l} \mathrm{e}^{-r^{2} / a^{2}-i l \phi}\left(\frac{r \sqrt{2}}{a}\right)^{|l|} L_{p}^{|l|}\left(\frac{2 r^{2}}{a^{2}}\right)
$$

where $p$ is the radial mode number, $a$ is a spot size parameter and $C$ is a normalization constant such that $\sum_{p, l}\left|a_{p, l}\right|^{2}=1$. LG modes are a convenient working basis, as they are eigenmodes of free space paraxial waves that carry OAM (ref. 2). The expansion was carried out on the fields propagated back to the undulator, where they also provide a straightforward map of the coherent helical microbunching structure. Figure 3 shows that $85 \%$ of the relative radiation power is carried by the primary $l=1$ OAM mode. The identical $l$-mode power distribution is also observed in the field decompositions at each camera plane, as one expects from conservation of angular momentum.

That the radiation contains OAM was further confirmed experimentally from the diffraction pattern generated as the radiation passes through a $200 \mu \mathrm{m}$ wide vertical slit. Shown in Fig. 4, the slit was centred on the intensity null, and the pattern observed by camera 2. The phase of an OAM mode on either horizontal side of the slit centred on the singularity is different by $\pi$, resulting in destructive interference along the axis and an intensity null in the observed diffraction pattern. If, on the other hand, the ring-like intensity were due to purely radial modes with no vortex (for example, from detuning), the light would be in-phase on both sides of the slit and produce a constructive interference on axis. By inspection of Fig. 4c, no intensity is observed on axis, consistent with the presence of a vortex. This conclusion is supported by comparison of the measured diffraction pattern with the similar pattern calculated from the reconstructed fields (Fig. 4b). For contrast, the pattern calculated with the phase vortex artificially removed (Fig. 4d) displays the on-axis peak inconsistent with a vortex. We note further that the observed shearing of the upper and lower bright spots likewise indicates the presence of a vortex with transverse energy flow ${ }^{28}$.

\section{Conclusions}

We expect that this approach can be readily extended to higher beam energies and shorter wavelength FELs, where the helical structure is more robust to transverse mixing and mode pollution ${ }^{4}$. Furthermore, the seed laser could be provided by the FEL itself, as in the recently demonstrated self-seeded configuration for hard $\mathrm{X}$-rays ${ }^{29}$. The concept may also be expanded to more advanced harmonic seeding schemes ${ }^{30}$ where both the frequency and OAM mode number can be strongly up-converted to generate highorder OAM light at short wavelengths. Extrapolation to these regimes must also take into account the deleterious effects of intra-beam scattering on the helical structure, and will be the subject of future studies.

Received 6 May 2013; accepted 1 July 2013; published online 18 August 2013

\section{References}

1. McNeil, B. W. J. \& Thompson, N. R. X-ray free-electron lasers. Nature Photon. 4, 814-821 (2010).

2. Allen, L., Beijersbergen, M. W., Spreeuw, R. J. C. \& Woerdman, J. P. Orbital angular momentum of light and the transformation of Laguerre-Gaussian laser modes. Phys. Rev. A 45, 8185-8189 (1992).

3. Hemsing, E. et al. Helical electron-beam microbunching by harmonic coupling in a helical undulator. Phys. Rev. Lett. 102, 174801 (2009).

4. Hemsing, E., Marinelli, A. \& Rosenzweig, J. B. Generating optical orbital angular momentum in a high-gain free-electron laser at the first harmonic. Phys. Rev. Lett. 106, 164803 (2011).

5. Spalding, G., Courtial, J. \& Leonard, R. D. Structured Light and Its Applications: An Introduction to Phase-Structured Beams and Nanoscale Optical Forces 1st edn (Academic, 2008)

6. He, H., Friese, M. E. J., Heckenberg, N. R. \& Rubinsztein-Dunlop, H. Direct observation of transfer of angular momentum to absorptive particles from a laser beam with a phase singularity. Phys. Rev. Lett. 75, 826-829 (1995).

7. Jesacher, A., Fürhapter, S., Bernet, S. \& Ritsch-Marte, M. Shadow effects in spiral phase contrast microscopy. Phys. Rev. Lett. 94, 233902 (2005).

8. Jack, B. et al. Holographic ghost imaging and the violation of a Bell inequality. Phys. Rev. Lett. 103, 083602 (2009).

9. Tabosa, J. W. R. \& Petrov, D. V. Optical pumping of orbital angular momentum of light in cold cesium atoms. Phys. Rev. Lett. 83, 4967-4970 (1999).

10. Fickler, R. et al. Quantum entanglement of high angular momenta. Science 338, 640-643 (2012).

11. Wang, J. et al. Terabit free-space data transmission employing orbital angular momentum multiplexing. Nature Photon. 6, 488-496 (2012).

12. Thole, B. T., Carra, P., Sette, F. \& van der Laan, G. X-ray circular dichroism as a probe of orbital magnetization. Phys. Rev. Lett. 68, 1943-1946 (1992).

13. Van Veenendaal, M. \& McNulty, I. Prediction of strong dichroism induced by X rays carrying orbital momentum. Phys. Rev. Lett. 98, 157401 (2007).

14. Beijersbergen, M. W., Allen, L., Van der Veen, H. E. L. O. \& Woerdman, J. P. Astigmatic laser mode converters and transfer of orbital angular momentum. Opt. Commun. 96, 123-132 (1993).

15. Heckenberg, N. R., McDuff, R., Smith, C. P., Rubinsztein-Dunlop, H. \& Wegener, M. J. Laser beams with phase singularities. Opt. Quant. Electron. 24, S951-S962 (1992).

16. Cai, X. et al. Integrated compact optical vortex beam emitters. Science 338, 363-366 (2012).

17. Beijersbergen, M. W., Coerwinkel, R. P. C., Kristensen, M. \& Woerdman, J. P. Helical-wavefront laser beams produced with a spiral phaseplate. Opt. Commun. 112, 327-327 (1994).

18. Bazhenov, V., Vasnetsov, M. \& Soskin, M. Laser-beams with screw dislocations in their wave-fronts. JETP Lett. 52, 429-431 (1990).

19. Peele, A. G. et al. Observation of an X-ray vortex. Opt. Lett. 27, 1752-1754 (2002).

20. Takahashi, Y. et al. Bragg X-ray ptychography of a silicon crystal: Visualization of the dislocation strain field and the production of a vortex beam. Phys. Rev. B 87, $121201(2013)$

21. Jentschura, U. D. \& Serbo, V. G. Generation of high-energy photons with large orbital angular momentum by Compton backscattering. Phys. Rev. Lett. 106, 013001 (2011).

22. Sasaki, S. \& McNulty, I. Proposal for generating brilliant X-ray beams carrying orbital angular momentum. Phys. Rev. Lett. 100, 124801 (2008).

23. Hemsing, E. et al. Experimental observation of helical microbunching of a relativistic electron beam. Appl. Phys. Lett. 100, 091110 (2012). 
24. Xiang, D. et al. Demonstration of the echo-enabled harmonic generation technique for short-wavelength seeded free electron lasers. Phys. Rev. Lett. 105, 114801 (2010).

25. Xiang, D. et al. Evidence of high harmonics from echo-enabled harmonic generation for seeding X-ray free electron lasers. Phys. Rev. Lett. 108, 024802 (2012)

26. Marinelli, A. et al. Single-shot coherent diffraction imaging of microbunched relativistic electron beams for free-electron laser applications. Phys. Rev. Lett. 110, 094802 (2013).

27. Allen, L. \& Oxley, M. Phase retrieval from series of images obtained by defocus variation. Opt. Commun. 199, 65-75 (2001).

28. Ghai, D. P., Senthilkumaran, P. \& Sirohi, R. Single-slit diffraction of an optical beam with phase singularity. Opt. Lasers Eng. 47, 123-126 (2009).

29. Amann, J. et al. Demonstration of self-seeding in a hard-X-ray free-electron laser. Nature Photon. 6, 693-698 (2012)

30. Hemsing, E. \& Marinelli, A. Echo-enabled X-ray vortex generation. Phys. Rev. Lett. 109, 224801 (2012).

\section{Acknowledgements}

The authors would like to acknowledge funding supported by US DOE under Contract Nos. DE-AC02-76SF00515 and DE-FG02-07ER46272.

\section{Author contributions}

E.H. conceived the theoretical concept and designed the experimental technique. E.H., A.K. and M.D. carried out the experiment. A.K. designed and built the helical undulator. A.M. contributed to the theoretical work and data analysis. D.X., J.B.R. and C.H. provided guidance on the experiment and on potential applications. All authors contributed to writing the paper.

\section{Additional information}

Reprints and permissions information is available online at www.nature.com/reprints. Correspondence and requests for materials should be addressed to E.H.

\section{Competing financial interests}

The authors declare no competing financial interests. 